\title{
Autoimmune disease drug discovery
}

\author{
Aris Persidis
}

Autoimmune disorders occur when the body's immune system turns against the body itself, attacking it as if it were a foreign pathogen. They comprise more than 50 distinct diseases and syndromes, and affect about $5 \%$ of the population in Europe and North America, with two thirds of the patients being female ${ }^{1}$. Examples of autoimmune disorders include rheumatoid arthritis, multiple sclerosis, juvenile diabetes, cardiomyopathy, antiphospholipid syndrome, Guillain-Barré syndrome, Crohn's disease, Graves' disease, Sjogren's syndrome, alopecia, myasthenia gravis, lupus erythematosus, and psoriasis. In addition, such disorders complicate other diseases that are not autoimmune in origin, such as Duchenne's muscular dystrophy and artherosclerosis.

The total societal disease burden for autoimmune disorders is difficult to estimate because while some of these are chronic and debilitating diseases, others are less serious. Arthritis alone is estimated to cause a $\$ 65$ billion disease burden ${ }^{2}$, so autoimmune disorders as a group are among the most expensive diseases faced by society today. As a result, they are the subject of significant research in both academic and industrial laboratories.

\section{Historical perspective}

Although the clinical manifestations of autoimmune diseases have long been known, the concept that the body's own immune system was responsible did not become established until reproducible experimental evidence began to be obtained from the mid1960s onward. For example, auto-antibodies against diseased organs in Sjogren's sydrome were first described in $1965^{3}$. Animal models at the time also showed how antibodies could be formed against red blood cells, suggesting correlations with some anemias ${ }^{4}$. The mid1960s also saw initial connections being made between viral infections and autoimmune diseases, specifically hemolytic anemia, based on clinical observations ${ }^{5}$.

In the early 1970s, autoimmune heart disease was first linked to $\alpha$-hemolytic streptococcus ${ }^{6}$, and the first theories were developed linking aging and autoimmuni$\mathrm{ty}^{7}$. Some autoimmune diseases also began to be described in increasing molecular

Aris Persidis is managing director of RheoGene, 706 Forest Street, Charlottsville, VA 22903 (apersidis@rheogene.com). detail. For example, diagnostic correlations were made between molecular markers, such as $\beta 2$ microglobulin concentration in saliva, and local inflammation in Sjogren's syndrome, suggesting how it was possible to identify specific molecules that were involved in the development of an autoimmune disease, and could therefore serve as diagnostics ${ }^{8}$. By the early 1980s, there were significant concerns about the links between bone marrow transplantation-a leadingedge procedure at the time-and autoimmune disease side effects 9 . By the early 1990s there was an interesting application of autoimmune disease principles in a novel direction, namely that of birth control by immunization against sperm ${ }^{10}$.

The last decade has also benefited from the advances of molecular biology and other techniques that enabled the identification of specific autoantigens involved in a variety of autoimmune disorders. This was critical for strategies to combat these disorders effectively, by focusing on the triggers and actual molecules that were involved. Significant progress has been made in these molecular approaches by using appropriate animal models. One example is autoimmune encephalomyelitis, a debilitating brain disease. Here, treatment of animal models of the disease with a neuroantigen and an antibody against CD11a (LFA-1) protected the ani- mals against the disease, showing that with appropriate molecular intervention, it was possible to divert the immune response from a destructive to a nondestructive response ${ }^{11}$. These efforts and others have resulted in a variety of therapeutic approaches against autoimmune disorders that continues to be refined.

\section{Current state}

Since autoimmune disorders cover such a wide variety of molecular and clinical phenomena, it should come as no surprise that research efforts span the gamut of approaches and therapeutic targets. The increasing understanding of the molecular basis of these diseases is helping to focus research directions in specific areas. Table 1 shows a selection of companies active in the development of autoimmune disorder therapeutics. The approaches range from gene therapy to antisense to administration of antibodies, vaccines, and a variety of cytokines.

At present, the advent of large amounts of genomic information is enabling the systematic attempt at correlating homologies between pathogens, such as viruses and bacteria, and specific self proteins. The hypothesis is that these pathogens might trigger an autoimmune response by virtue of these homologies. For example, the novel thyroid

Table 1. Selected companies with autoimmune drug discovery programs.

\begin{tabular}{|c|c|}
\hline Company & Program \\
\hline Alexion (New Haven, CT) & Monoclonal antibody for lupus \\
\hline Anergen (Redwood City, CA) & Peptide vaccine for rheumatoid arthritis \\
\hline Biogen (Cambridge, MA) & Anti-CD40 antibody for lupus \\
\hline Centocor (Malvern, PA) & Chimeric anti-TNF antibody for rheumatoid arthitis \\
\hline Chiron (Emeryville, CA) & Anti-TNF antiobody for multiple sclerosis \\
\hline Connetics (Palo Alto, CA) & Recombinant relaxin for scleroderma \\
\hline IDEC Pharmaceuticals (San Diego, CA) & $\begin{array}{l}\text { Monoclonal antibodies for rheumatoid arthritis } \\
\text { and lupus }\end{array}$ \\
\hline Immune Response Corp. (Carlsbad, CA) & Vaccine for rheumatoid arthritis \\
\hline Immunex (Seattle, WA) & Recombinant TNF receptor for rheumatoid arthritis \\
\hline Isis Pharmaceuticals (Carlsbad, CA) & Antisense for rheumatoid arthritis \\
\hline Medarex (Annandale, $\mathrm{NJ}$ ) & $\begin{array}{l}\text { Monoclonal antibody for idiopathic thrombocy- } \\
\text { topenic purpura }\end{array}$ \\
\hline Neurocrine Biosciences (San Diego, CA) & Cytokines for autoimmune diseases \\
\hline Ortho Biotech (Raritan, NJ) & $\begin{array}{l}\text { Monoclonal antibody for CD4-mediated } \\
\text { autoimmune diseases }\end{array}$ \\
\hline Protein Design Labs (Mountain View, CA) & Monoclonal antibody for autoimmune diseases \\
\hline Schering-Plough (Madison, NJ) & IL-10 for rheumatoid arthritis \\
\hline Seragen (Hopkinton, MA) & IL-2 fusion protein for severe rheumatoid arthritis \\
\hline
\end{tabular}

Source: Biovista (www.biovista.com). 
autoantigen that is the sodium/iodide symporter (NIS) has local amino acid sequence homologies with three other known thyroid autoantigens, namely thyroglobulin, thyroid peroxidase, and thyrotropin receptor. A recent report describes the application of a computer-aided search that showed how the NIS was found to have significant local homologies with no fewer than other 11 proteins from bacteria or viruses, such as Streptococcus or herpes. It was also found to have extensive-but not local-homologies with several unknown proteins from invertebrates such as Drosophila melanogaster and Caenorhabditis elegans, and with bacteria such as Bacillus subtilis and Xanthobacter ${ }^{12}$.

Animal models are being used extensively to discover and confirm antigens involved in autoimmune disorders, and also to help develop effective therapies based on that knowledge. A recent study describes how rats were induced to have a particular version of myasthenia by injecting them with peptides derived from the voltage-gated calcium channel found in neuromuscular junctions ${ }^{13}$. This protein is believed to be the target of autoantibodies found in patients with this disease, and the rat model is helping to characterize the specific epitopes of the protein that are recognized by these antibodies. Such knowledge can then be used to tailor a vaccine or other therapeutic modality to interfere with and perhaps abrogate this autoimmune response.

\section{Industry challenges}

A key challenge to developing therapies for autoimmune disorders is the cross-reactivity between normal body proteins and those of a variety of pathogenic agents. For example, hepatitis B virus has an amino acid sequence in one of its proteins that is highly homologous to that of myelin basic protein (MBP), which is the target of autoimmune attack in multiple sclerosis. Brain inflammation is caused in animal models injected with the MBP-homologous component of the hepatitis B virus. Adenovirus type 2 is another virus with a protein that has significant sequence homology to MBP. Another example is rheumatoid arthritis. In animal models, inflammation of the joints can be caused by infection with Mycobacterium tuberculosis, which has protein components similar to normal cartilage. Finally, rheumatic fever is sometimes accompanied by damage to heart valves. Animal models have shown the connection between such damage and hemolytic streptococcus, which has a cell wall protein similar to heart muscle myosin. Determining all of these sequence similarities that are not by-products of evolutionary conservation but that actually have a causative effect in autoim- munity is a major challenge to researchers. It is being addressed by systematic analysis and sequence comparison derived from genomics coupled with relevant clinical information.

Another challenge is identifying not only those autoantigens that are shared with pathogens, but also those that are part of the native body itself, and that trigger disease as a result of as yet poorly understood events. A good example of new classes of such autoantigens is DNA itself. A recent report highlights the identification and characterization of antibodies against DNA itself, which might lead to numerous complications associated with cell division and gene expression $^{14}$.

\section{New approaches tend to employ therapies based on immunomodulation, rather than immunosup- pression, and in the case of multiple sclerosis, these approaches are proving to be more effective.}

\section{Future directions}

As our understanding of the molecular and cellular aspects of autoimmunity increases, we will continue to see more effective treatments for these diseases. For example, multiple sclerosis (MS) is an autoimmune neurological disorder thought to be mediated by antigen-specific CD4+ T helper (Th1) T-cells which cause demyelination of the central nervous system (CNS). Many current therapeutic strategies attempt to downregulate the entire immune system by causing generalized immunosuppression, in the hope that this will reduce the specific action of the T-cells involved. This approach, incidentally, is widespread in autoimmune treatments in the absence of better options. Unfortunately, generalized immunosuppression has not met with the success expected. Now, new approaches tend to employ therapies based on immunomodulation, rather than immunosuppression, by administration of cytokines such as interferon (IFN) $\beta$ and glatiramer acetate, and in the case of MS, these approaches are proving to be more effective ${ }^{15}$. Refinements in our understanding of the effects of immunomodulation versus more drastic measures will no doubt help us devise even more effective therapies.

The future will also see the identification of putative autoantigens occur at an increasing rate, based on the application of funtion- al genomics techniques. For example, a new MHC-restricted autoantigen from the insulin B chain was recently identified in an animal model of type I diabetes by screening a disease and pancreatic-specific cDNA library ${ }^{16}$. This epitope was found to be the same one recognized by pathogenic CD4+ Tcells, and may thus be formulated into an insulin-derived preventive therapy.

We are also likely to see major efforts being undertaken to explain the gender difference that is observed in susceptibility to autoimmune disease. There are hypotheses that involve hormonal and other differences, but at this point many questions have yet to be answered ${ }^{17}$.

Finally, the future will also see the application of gene therapy approaches to the treatment of autoimmune disorders, based on the administration of genes or mini-genes encoding for immunomodulatory cytokines, or antigens that would cause tolerization. Efforts are already underway with administration of antiinflammatory cytokines such as transforming growth factor- $\beta 1$ and interleukin 4 , which had protective effects in animal models of experimental allergic encephalomyelitis ${ }^{18}$.

\section{Conclusions}

Autoimmune disorders are a family of diseases that represent a major societal burden. While the pace of our understanding of the molecular and cellular processes of these complex disorders have thus far been behind that of other, simpler diseases, the advent of large-scale genomic and functional analysis tools are now helping redress the balance, and the enormous pressure to understand these diseases and cure them is the best guarantee of progress in this area.

\footnotetext{
American Autoimmune Related Diseases Association (www.aarda.org).

2. Persidis, A. Nat. Biotechnol. 17, 726-728 (1999).

3. Bertram, U. \& Halberg, P. Acta Allergol. 20, 472-483 (1965).

4. Sundermann, A. \& Mey, U. Folia Haematol. Int. Mag. Klin. Morphol. Blutforsch. 84, 387-401 (1965).

5. Puxeddu, A. et al. Haematologica 50, 1073-1092 (1965).

6. Wachsmuth, E.D. \& Born, U. Br. Med. J. 3, 623 (1972).

7. Bastenie, P.A Bull. Mem. Acad. R. Med. Belg. 130, 517-526 (1975).

8. Michalski, J.P. et al. N. Engl. J. Med. 293, 1228-1231 (1975).

9. Minchinton, R.M. et al. Lancet 2, 391 (1982)

10. De Almeida, M. et al. Hum. Reprod. 6, 405-410 (1991).

11. Willenborg, D.O. et al. J. Immunol. 157, 1973-1980 (1996).

12. Benvenga, S. et al. J. Endocrinol. Invest. 22, 535-540 (1999).

13. Komai, K. et al. J. Neurol. Sci. 166, 126-130 (1999).

14. Eilat, D. \& Naparstek, Y. Immunol. Today 20, 339-342 (1999).

15. Giovannoni, G. \& Miller, D.H. J. R. Coll. Physicians Lond. 33, 315-322 (1999).

16. Wong, F.S. et al. Nat. Med. 5, 1026-1031 (1999)

17. Nikezic-Ardolic, M. et al. Lupus 8, 375-379 (1999).

18. Piccirillo, C.A. \& Prud'homme, G.J. Hum. Gene Ther. 10, 1915-1922 (1999).
} 